\title{
Malignant Hypopharyngeal Neoplasm
}

National Cancer Institute

\section{Source}

National Cancer Institute. Malignant Hypopharyngeal Neoplasm. NCI Thesaurus. Code C7190.

A primary or metastatic malignant neoplasm that affects the hypopharynx. 